\title{
A Sub-Zonal PMV-Based HVAC and Façade Control System for Curtain Wall Buildings ${ }^{\dagger}$
}

\author{
Marco Arnesano 1,*, Andrea Calvaresi ${ }^{1}$, Filippo Pietroni ${ }^{1}$, Lorenzo Zampetti ${ }^{1}$, Sara Magnani ${ }^{2}$, \\ Oscar Casadei ${ }^{2}$ and Gian Marco Revel ${ }^{1}$ \\ 1 Department of Industrial Engineering and Mathematical Sciences, Università Politecnica delle Marche, \\ via Brecce Bianche 12, 60131 Ancona, Italy; a.calvaresi@pm.univpm.it (A.C.); f.pietroni@univpm.it (F.P.); \\ l.zampetti@univpm.it (L.Z.); gm.revel@univpm.it (G.M.R.) \\ 2 Focchi Spa, via Cornacchiara, 47824 Poggio Torriana (RN), Italy; s.magnani@focchi.it (S.M.); \\ o.casadei@focchi.it (O.C.) \\ * Correspondence: m.arnesano@univpm.it; Tel.: +39-071-2204508 \\ † Presented at the Sustainable Places (SP 2018), Aix-les Bains, France, 27-29 June 2018.
}

Published: 27 August 2018

\begin{abstract}
This paper presents an experimental test room in a curtain wall building where an innovative monitoring and control system was implemented and tested. The proposed solution is composed by an IR-based comfort sensor that measures the PMV (Predicted Mean Vote) index for 2 room's sub-zones and provides the optimal air temperature set-points. The overall control system includes a distributed sensors and actuators network, also embedded into the façade modules, to measure indoor and outdoor parameters and to regulate fan-coils, windows opening and shadings with a sub-zonal approach. Initial results turn out to provide an energy saving of about $20 \%$ with an improvement of thermal/visual comfort and IAQ conditions.
\end{abstract}

Keywords: monitoring; façade; HVAC; comfort; energy; optimization

\section{Introduction}

The energy is mostly used in buildings to guarantee occupants' comfort. In fact, about $40 \%$ of the energy is used for heating, cooling and ventilation [1] and up to $30 \%$ of this consumption is accounted to glazed surface [2], the major part of curtain wall buildings. Previous experiences demonstrated that the implementation of smart facades systems leads to significant energy savings $[3,4]$. Such savings can be achieved because of the capability of controlling the incoming solar radiation, which is one of the most influencing quantity of the building energy balance, thermal and visual comfort. To optimize the control of the HVAC and façade systems, the fine-grained measurement of the radiant temperatures is required. An example was presented in [5] where an innovative IR-based thermal comfort sensor [6] was used to measure thermal comfort in two subzones of an office room to control two heaters separately and to provide heat only where needed because of the different comfort requirements caused by the glazed surface. This work presents the application of that approach to a meeting room in a curtain wall building and integrated with a BMS to apply the sub-zonal control of the HVAC and façade systems.

\section{Materials and Methods}

\subsection{General Description of the Proposed System}

The basic idea of the proposed solution is to measure and control separately different sub-zones of a room to regulate the heating/cooling and illumination according to local needs that depend on 
the local outdoor and indoor conditions. The sub-zonal breakdown is done according to the room dimensions, walls orientation, HVAC and lighting grouping possibilities. Once that the breakdown is decided, at least one façade module, equipped with sensors, is assigned to each sub-zone. If a subzone includes more than one orientation (e.g., corner), one sensing façade module for each orientation has to be included. The sensing façade module includes sensors to measure outdoor and indoor conditions. Following the same approach, the indoor thermal conditions have to be measured with the same granularity, which is not feasible using the traditional thermostat. For this reason, the methodology described in [5], based on the Comfort Eye sensor [6], has been applied. The sensing facades and Comfort Eye are then integrated into a BMS system with embedded software that allows the optimal control. Each component and the architecture are described in the following sections.

\subsection{Description of the Case Study}

The proposed approach was implemented in a meeting room $(12 \times 5.70 \times 3 \mathrm{~m})$ of the headquarter of Focchi S.p.A., located in the industrial area of Poggio Torriana (RN), in central Italy (latitude: $44^{\circ} 05^{\prime} \mathrm{N}$, Longitude: $12^{\circ} 41^{\prime} \mathrm{E}$, altitude: $52.90 \mathrm{~m}$ ). The region is characterized by a hot summer Mediterranean climate. The test room is characterized by a continuous glazed facade on 3 sides. The room was virtually divided into two sub-zones, one is South-oriented and the other one is North-oriented. Following the methodology described before, two Comfort Eye systems were installed (one in each sub-zone) together with 4 façade modules with embedded sensors. The heat and cool is provided by a 2-pipes circuit with 7 fancoils that have been grouped according to the sub-zonal breakdown. The general scheme of the test room is shown in Figure 1.

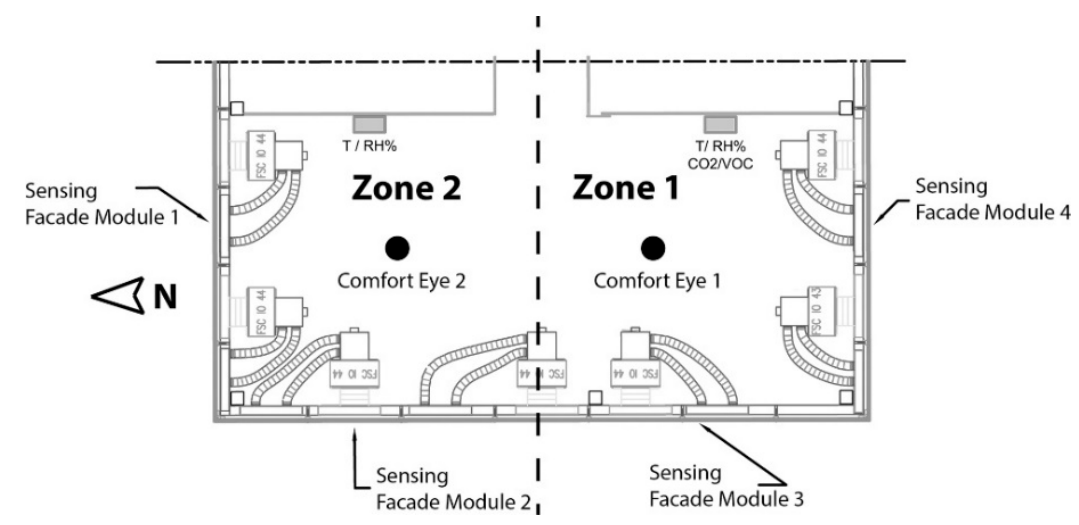

Figure 1. Scheme of the test room.

\subsection{Monitoring and Control Architecture}

\subsubsection{Comfort Eye}

The monitoring of thermal comfort is provided by the innovative Comfort Eye sensor. As presented in [6] the concept of the Comfort Eye is based on an IR scanning system, installed on the ceiling of the room to measure the indoor surface temperatures. The thermal maps are sent to a gateway, which calculates the mean radiant temperature and, using the data from environmental sensors (i.e., air temperature, relative humidity), the Comfort Eye provides the PMV index. The calculations are performed for different positions of the room, near and far from the glazed surfaces, and for typical room occupants' profiles: the metabolic rate is set equal to 1.2 met and the clothing level calculated on daily basis in function of the outdoor temperature. According to ISO 7726 and ISO 7730, thermal comfort parameters are estimated for several sub-zones of the environment and a root finding algorithm is included in the embedded software to calculate the air temperature which allows the highest level of comfort $(\mathrm{PMV}=0)$ in each sub-zone. This air temperature values are provided as output to be used as setpoint for the HVAC management. 


\subsubsection{Façade Module with Embedded Sensors}

A new façade module was developed to integrate natively sensors and actuators, with dedicated housing for hardware and cabling. The modules are composed by double pane windows and aluminum frame with thermal break. Each sensing modules is equipped with the following sensors:

- Outdoor: solar radiation and illuminance on the vertical plan

- Indoor: solar radiation and illuminance on the horizontal plan

The modules all, with and without sensors, include actuators to regulate the shading position and the window opening.

\subsubsection{Building Management System}

A dedicated Building Management System was developed to integrate all sensors and actuators in a unique system and to apply control logics. Figure 2 illustrates the architecture of the BMS developed to implement the system. Thermal comfort, visual comfort and indoor air quality control logics were developed to provide the optimal control of the room. The control flows were developed with the sub-zonal approach: each sub-zone is controlled taking into account the indoor and outdoor conditions measured in that sub-zone. Finally, the control logics were developed balancing the different comfort requirement while maximizing the energy efficiency.

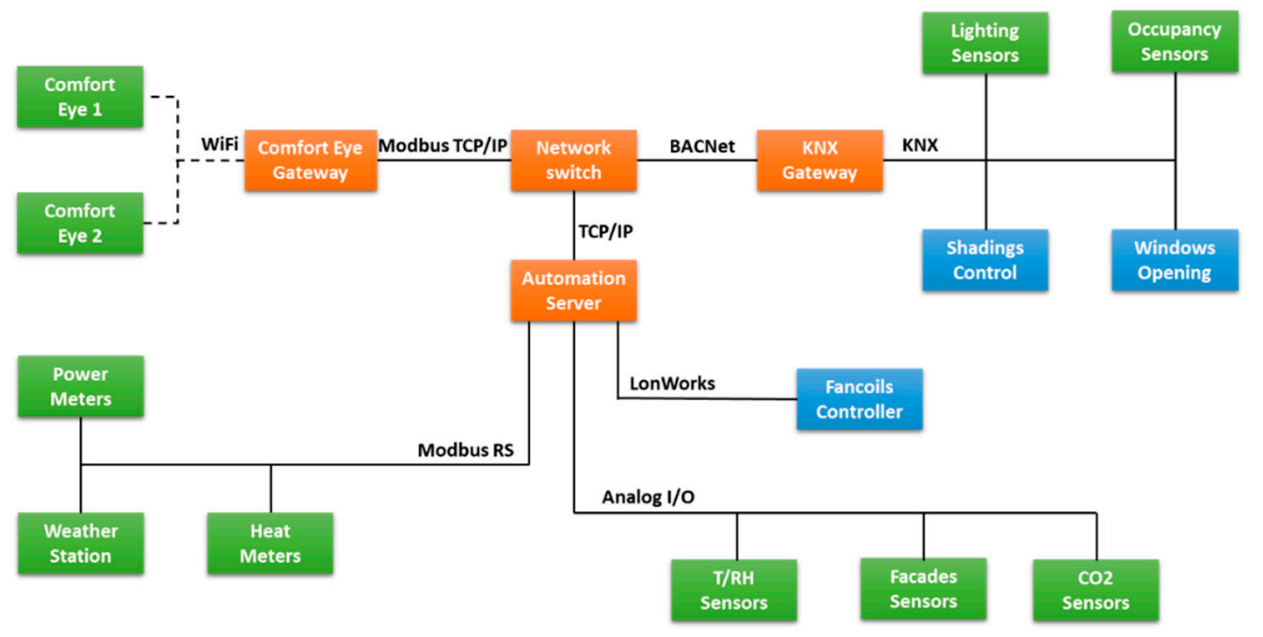

Figure 2. Architecture of the developed BMS.

\section{Results}

The integration of the Comfort Eye with the BMS and facade aims at maximizing thermal comfort with the minimum energy consumption. A preliminary analysis of performance has been conducted comparing two scenarios in cooling season:

1. System ON: the BMS controls the HVAC making use of the real-time temperature setpoints provided by the Comfort Eye and the façade modules controlled according to the logics described above;

2. System OFF: the BMS replicates the room operation before the installation of the sub-zonal solution. Temperature setpoint fixed at $26^{\circ} \mathrm{C}$ in all sub-zones, fixed fans velocity and manual operation of windows, shadings and lights.

Two working days have been compared: 4 August 2017 with System ON (outdoor average air temperature: $35.1{ }^{\circ} \mathrm{C}$ ) and 20 July 2018 with System OFF (outdoor average air temperature: $30.3^{\circ} \mathrm{C}$ ). Results for thermal energy consumption are reported in Figure 3. The test turned out to provide a total energy saving of the $22 \%$, calculated only in the occupied hours (10-13 with red box). The higher saving is achieved in the Zone 2 (North Oriented) where the Comfort Eye assigned a higher setpoint given the lower mean radiant temperature (colder surfaces). During both days the system was able to 
keep comfort with the optimal range \pm 0.5 PMV. To be noticed that the System OFF registered negative PMV values (toward cold sensation), mostly in the Zone 2 (North oriented), which is symptom of a non-optimal comfort management that cannot balance the different thermal conditions of the room.
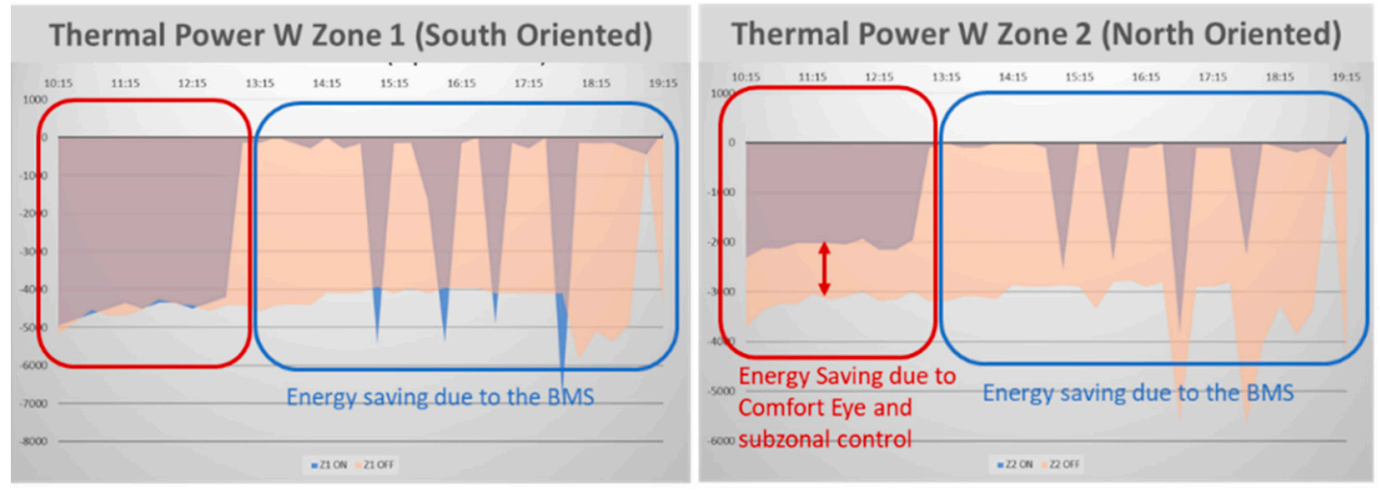

Figure 3. Comparison of thermal energy with and without the proposed solution.

\section{Conclusions}

This work presented the experimental testing of an innovative solution for the implementation of sub-zonal room control for curtain wall buildings. The main goal of the system is to provide finegrained HVAC and windows management according to local conditions that are mainly influenced by the incoming solar radiation. This is particularly important for curtain walls buildings where the envelope is primarily composed by glazed surface. To achieve the proposed objective, a monitoring system capable of measuring the local outdoor and indoor conditions was set up. The system is composed by the Comfort Eye sensors and sensing façade modules, integrated with a dedicated BMS. The application to a real room in a curtain wall building demonstrated that the proposed solution is effective and can provide an energy saving up to $20 \%$ in cooling season, maintaining occupants' comfort. These results have been achieved by controlling the room with different setpoints in the two sub-zones that have a different orientation, demonstrating the importance of controlling the space taking into account local needs. Further testing and validation sessions of the room will be done in the next future to consolidate the validation.

Funding: This research received no external funding.

Conflicts of Interest: The authors declare no conflict of interest.

\section{References}

1. Saheb, Y. Modernising Building Energy Codes to Secure Our Global Energy Future; IEA: Paris, France, 2013.

2. Arasteh, D.; Selkowitz, S.E.; Apte, J. Zero Energy Windows. In Proceedings of the ACEEE Summer Study on Energy Efficiency in Buildings, Pacific Grove, CA, USA, 13-18 August 2006.

3. Mingzhe, L.; Wittchen, K.B.; Heiselberg, P.K. Control strategies for intelligent glazed façade and their influence on energy and comfort performance of office buildings in Denmark. Appl. Energy 2015, 145, $43-51$.

4. Al Touma, A.; Ouahrani, D. Shading and day-lighting controls energy savings in offices with fully-Glazed façades in hot climates. Energy Build. 2017, 151, 263-274.

5. Zampetti, L.; Arnesano, M.; Revel, G.M. Experimental testing of a system for the energy-efficient sub-zonal heating management in indoor environments based on PMV. Energy Build. 2018, 166, 229-238.

6. Revel, G.M.; Arnesano, M.; Pietroni, F. Development and validation of a low-cost infrared measurement system for real-time monitoring of indoor thermal comfort. Meas. Sci. Technol. 2014, 25, doi:10.1088/09570233/25/8/085101.

(C) 2018 by the authors. Licensee MDPI, Basel, Switzerland. This article is an open access article distributed under the terms and conditions of the Creative Commons Attribution (CC BY) license (http://creativecommons.org/licenses/by/4.0/). 